\title{
Impact of the Mechanical Interface on BCG Signals Obtained from Electronic Weighing Scales
}

\author{
Ramon Casanella, Joan Gomez-Clapers, Marc Hernandez-Urrea, Ramon Pallas-Areny \\ Universitat Politècnica de Catalunya - BarcelonaTech, Castelldefels, Spain
}

\begin{abstract}
This work analyzes the reproducibility of the BCG obtained from different weighing scales. First, the natural frequency of three commercial bathroom scales has been characterized by an impulse excitation test. Second, the $B C G$ and the ECG from 5 healthy subjects ( $27 \pm 4$ years) have been recorded for $60 \mathrm{~s}$ by sequentially standing on these weighing scales connected to the same analog front-end and signal acquisition system. An ensemble average representative of each subject and weighing scale has been obtained by applying Woody's method with the ECG as a timing reference. Consecutive BCG signals obtained from the three scales show consistent systematic intra-subject differences in the timing of the $J$ peak that can reach up to $30 \mathrm{~ms}$. This timing error is relevant because it is about $50 \%$ of the changes induced by typical respiratory maneuvers, such as Valsalva's, used to modulate hemodynamic parameters in correlation studies between changes in $J$ timing with respect to different cardiac fiducial points. Due to its ubiquity, weighing scales are promising devices for monitoring cardiovascular function at home but their frequency response must be accounted for and minimal performance standards should be defined for them.
\end{abstract}

\section{Introduction}

The interest in periodic monitoring of cardiovascular information at home or in other non-clinical scenarios has increased in recent years. For that purpose, ballistocardiogram (BCG) systems have been embedded in beds, chairs or weighing scales, among others [1]. The BCG is the recording of forces in the body that result from cardiac ejection and several BCG parameters have been correlated to important cardiovascular markers such as heart rate [2], cardiac output [3], pre-ejection period [4] or systolic blood pressure changes [5] by using modified weighing scales as recording devices. In an early work that used weighing scales to acquire BCG signals [6], it was assumed that they were underdamped low-pass second-order mechanical systems with a natural frequency close to the signal bandwidth. Further, it was observed there that different weighing scales yielded different BCG waveforms for the same subject, albeit this was irrelevant for heart rate monitoring [2]. Unfortunately, these results went mostly unnoticed during later characterizations of weighing scales intended for BCG monitoring [7], which concluded that the measured waveform was not mechanically distorted as the resonant frequency of scales was significantly out of band. This view, in which the mechanic response of the weighing scale is considered not to distort the BCG signal, has been sustained until now $[1,8]$. Contrarily, the influence of the electronic interface has received more attention as a necessary step towards the standardization of electronic stages of BCG acquisition systems [9]. On the other hand, it has been recently pointed out that time correlations between the pre-ejection period and some features of the BCG are different when measured using a force plate or a weighing scale [10], which has been attributed to the wider bandwidth of force plates. This finding further increases the need of characterizing the mechanical interfaces used in BCG systems so that performance standards regarding both electronic and mechanical aspects could be established in the near future. This work aims to contribute to this goal by analyzing the reproducibility of the BCG obtained in different weighing scales and subjects.

\section{Materials and methods}

\subsection{Weighing scales and signal acquisition system}

The weighing scales used in this work were: WS1 (Seca sense 804), a bathroom scale with a plastic top surface; WS2 (Balance KH5510), a similar bathroom scale but with glass platform; and WS3 (MCR Electronic), a pharmacy body weight scale with rubbercovered metal surface.

The BCG was obtained from each scale by arranging their strain gauges to form a Wheatstone bridge that was connected to a typical BCG analog front-end [9]. This provided a total gain of 25,000 and a bandwidth from 
$0.5 \mathrm{~Hz}$ to $25 \mathrm{~Hz}$ that minimizes possible errors in the timing of BCG waves [9].

Additionally, a lead I ECG was recorded between hands by using a custom ambulatory ECG system [9], to be used as a timing reference during the analysis of BCG data.

The BCG and the ECG were acquired by a data acquisition system (MicroDAQ-Lite, Eagle Technology, Cape Town, South Africa) configured to sample each channel at $350 \mathrm{~Hz}$ and send the data to a PC, where the recordings were analyzed with $\mathrm{MATLAB}^{\circledR}$.

\subsection{Experimental setup}

In a first experiment, the $2^{\text {nd }}$ order dynamic properties of the weighing scales were determined from their response to an impulse excitation (Impulse Excitation Test, IET), performed as described in [2] in which a tennis ball was dropped over the scale platform (with zero initial velocity) and caught after its bounce.

Afterwards, the BCG and the ECG were recorded from 5 healthy subjects (see Table 1), which gave their informed consent, for $60 \mathrm{~s}$ sequentially in each of the three weighing scales connected to the same signal acquisition system described above.

Table 1. Cohort characteristics

\begin{tabular}{lllll}
\hline Subject & Gender & Age & Weight $(\mathrm{kg})$ & Height $(\mathrm{cm})$ \\
\hline S1 & F & 28 & 55 & 170 \\
S2 & M & 23 & 71 & 174 \\
S3 & F & 26 & 62 & 166 \\
S4 & M & 26 & 74 & 182 \\
S5 & M & 33 & 72 & 188 \\
\hline
\end{tabular}

An ensemble average representative of each subject and weighing scale was obtained by applying Woody's method [11] with the ECG as a timing reference.

\section{Results and discussion}

Figure 1 shows an example of the normalized traces obtained during an IET that were used to determine the mechanical responses of the three weighing scales that are summarized in Table 2.

Table 2. Mechanical properties of the weighing scales

\begin{tabular}{lcc}
\hline & Natural frequency $(\mathrm{Hz})$ & Damping ratio \\
\hline WS1 & 55 & 0.02 \\
WS2 & 28 & 0.07 \\
WS3 & 35 & 0.03 \\
\hline
\end{tabular}

As expected from their different constitutive materials, the mechanical properties of the three commercial devices are significantly different.

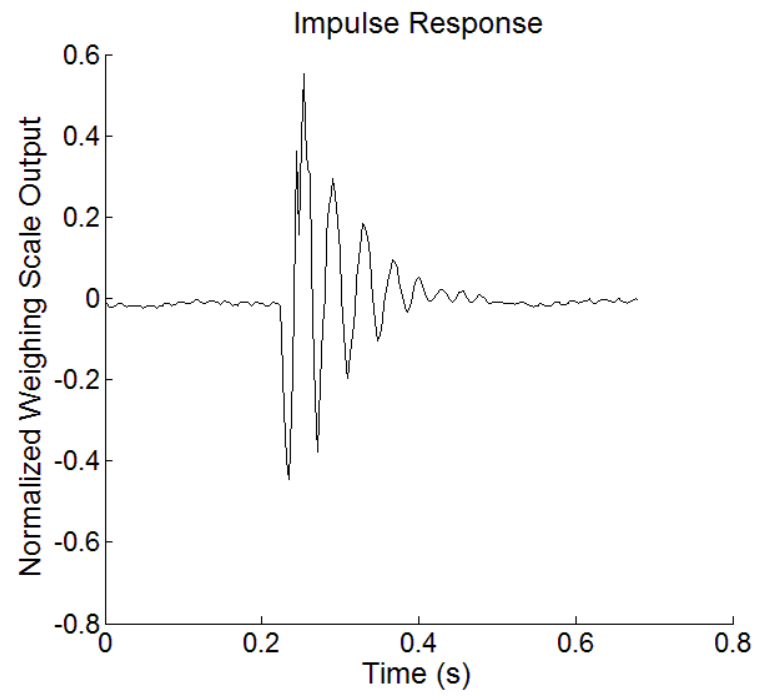

Figure 1. Example of the impulse response of WS1.

The effect of these different mechanical properties on the normalized BCG of each of the five subjects under study is shown in Figures 2 to 6.

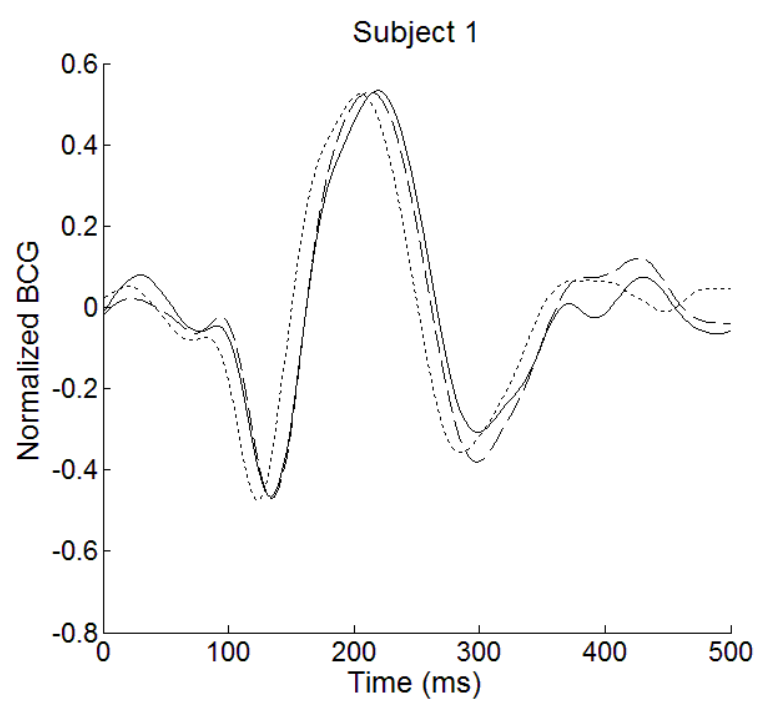

Figure 2. Ensemble averages of the three consecutive BCGs acquired using WS1 (dashed), WS2 (dotted), and WS3 (solid) for subject 1. 


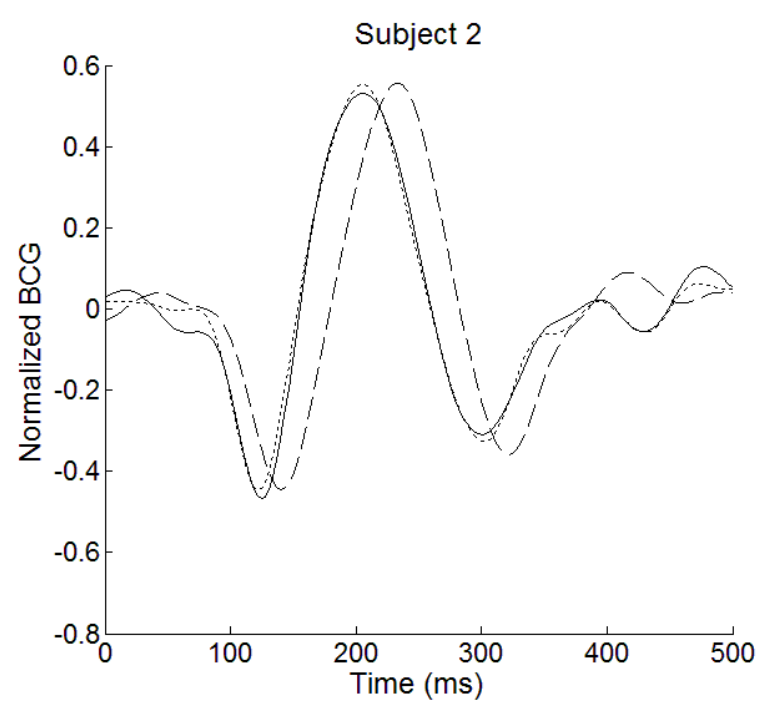

Figure 3. Ensemble averages of the three consecutive BCGs acquired using WS1 (dashed), WS2 (dotted), and WS3 (solid) for subject 2.

The results show that the effect of the weighing scales on the BCG depend on the subject and are specially relevant for S1 and S2, for which differences in the timing of the $\mathrm{J}$ peak (the most prominent positive peak in the center of the BCG wave) can reach up to $30 \mathrm{~ms}$. This is about $50 \%$ of the changes induced by typical respiratory maneuvers, such as Valsalva's, used to modulate hemodynamic parameters in correlation studies between changes in $\mathrm{J}$ timing with respect to different cardiac fiducial points $[4,5]$.

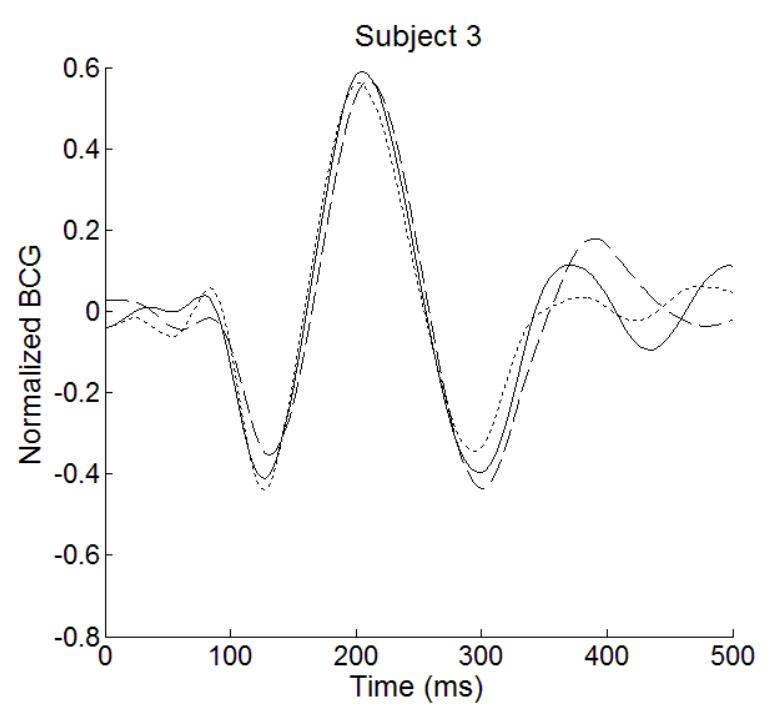

Figure 4. Ensemble averages of the three consecutive BCGs acquired using WS1 (dashed), WS2 (dotted), and WS3 (solid) for subject 3.

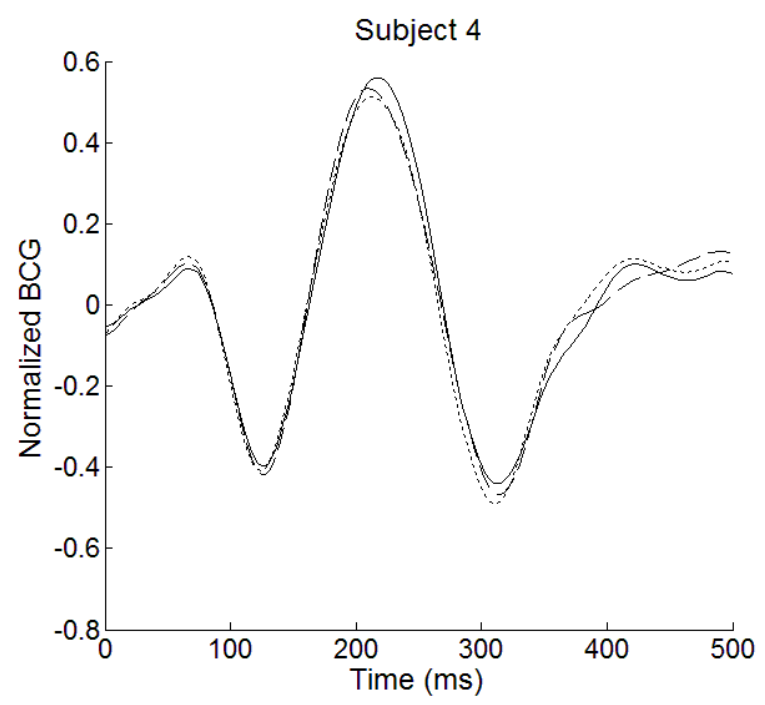

Figure 5. Ensemble averages of the three consecutive BCGs acquired using WS1 (dashed), WS2 (dotted), and WS3 (solid) for subject 4.

For S3, and especially for S4 and S5, the J-peak timing is less influenced by the weighing scales, but important waveform differences still exist, especially in the latter waves of the signal.

All these results suggest that in BCG's measured with commercial weighing scales there can be a non-negligible coupling effect between the mechanical response of the weighing scale and that of the subject, which depends not only on the mechanical properties of the scales but also on those of the subjects.

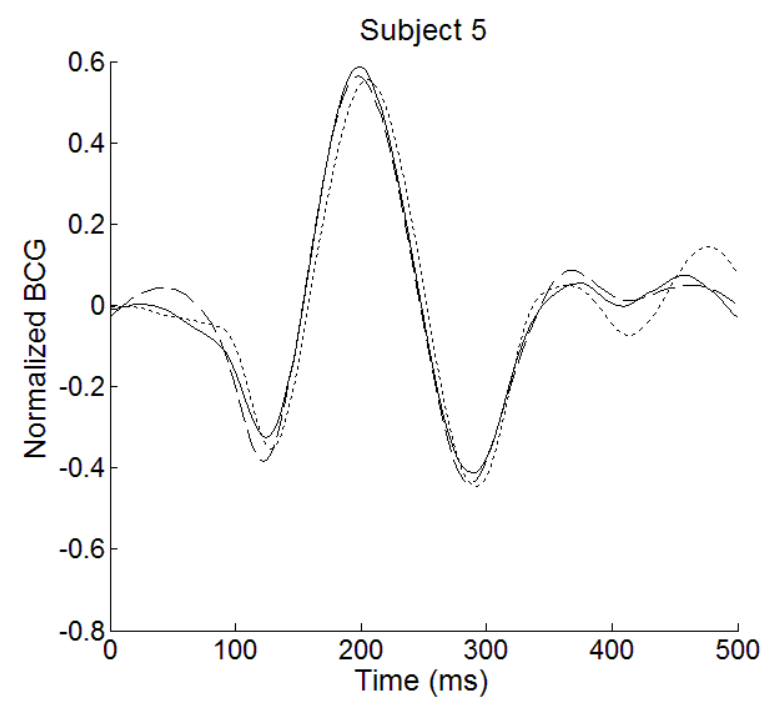

Figure 6. Ensemble averages of the three consecutive BCGs acquired using WS1 (dashed), WS2 (dotted), and WS3 (solid) for subject 5.

Finally, Figure 7 shows an example of the results obtained when acquiring two consecutive BCG's for the 
same subject in the same scale. The similarity between waveforms observed in these cases confirm that the existing differences in the signals are attributable to the differences in the mechanical properties of weighing scales and not to a possible lack of repeatability in the BCG signals acquired.

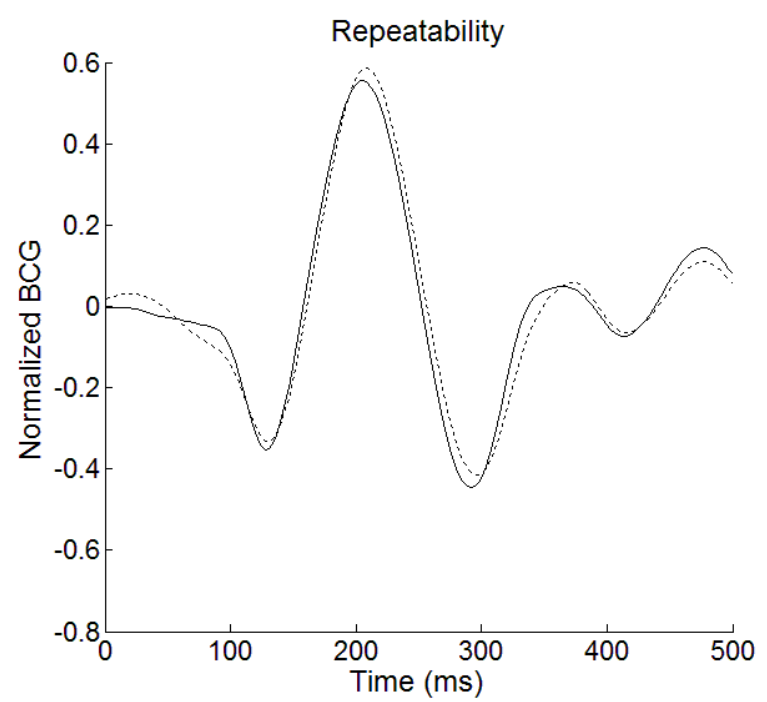

Figure 7. Ensemble averages of two consecutive BCGs acquired using WS2 for subject 5 to evaluate the repeatability of the waveform.

\section{Conclusions}

The reproducibility of the BCG obtained from different weighing scales and subjects has been analyzed. The results obtained show that the three commercial weighing scales analyzed have different mechanical responses that influence BCG waveforms to different extents depending on the subject and that for some of them the timing differences in the main waves of the waveform can be relevant. These very different effects for different subjects that can be detected even in the reduced cohort studied need to be carefully characterized for a larger cohort in the future in order to determine to what extent these can be corrected.

The BCG is indeed a very promising tool for unobtrusively monitoring cardiovascular parameters at home and in other non-clinical scenarios. Nevertheless, the impact on the signal of the mechanical interfaces through which BCG is currently recorded, such as those of beds, chairs and weighing scales needs still to be clearly determined. In the near future, an extensive work of characterization of the several existing mechanical interfaces is indeed required as a first step towards defining the necessary mechanical standards to be fulfilled by the forthcoming instruments in the field.

\section{Acknowledgements}

The authors want to thank the volunteers for their valuable cooperation and F. Lopez for his technical support.

\section{References}

[1] O. T. Inan, P. F. Migeotte, K.-S. Park, M. Etemadi, K. Tavakolian, et al. Ballistocardiography and Seismocardiography: A Review of Recent Advances. Biomed. Heal. Informatics, IEEE J. 2015; 19(4): 1414-27.

[2] R. Pallas-Areny, R. Gonzalez-Landaeta, O. Casas. Heart Rate Detection from an Electronic Weighing Scale. Physiol. Meas. 2008; 29(8): 979-88.

[3] O. T. Inan, M. Etemadi, A. Paloma, L. Giovangrandi, G. T. A. Kovacs. Non-invasive Cardiac Output Trending During Exercise Recovery on a Bathroom-scale-based Ballistocardiograph. Physiol. Meas. 2009; 30(3): 261-74.

[4] M. Etemadi, O. T. Inan, L. Giovangrandi, G. T. A. Kovacs. Rapid Assessment of Cardiac Contractility on a Home Bathroom Scale. Inf. Technol. Biomed. IEEE Trans. 2011; 15(6): 864-69.

[5] J. H. Shin, K. M. Lee, K. S. Park. Non-constrained Monitoring of Systolic Blood Pressure on a Weighing Scale. Physiol. Meas. 2009; 30(7): 679-93.

[6] R. Pallas-Areny, R. Gonzalez-Landaeta, and O. Casas. Heart Rate Detection from an Electronic Weighing Scale. Eng. Med. Biol. Soc. (EMBS), 2007. 29th Annu. Int. Conf. IEEE. 2007: 6282-5.

[7] O. T. Inan, M. Etemadi, R. M. Wiard, L. Giovangrandi, G. T. A. Kovacs. Robust Ballistocardiogram Acquisition for Home Monitoring. Physiol. Meas. 2009; 30(2): 169-85.

[8] A. D. Wiens and O. T. Inan. A Novel System Identification Technique for Improved Wearable Hemodynamics Assessment. Biomed. Eng. IEEE Trans. 2015; 62(5): 1345-54.

[9] J. Gomez-Clapers, A. Serra-Rocamora, R. Casanella, R. Pallas-Areny. Towards the Standardization of Ballistocardiography Systems for J-peak Timing Measurement. Measurement. 2014; 58:310-16.

[10] H. Ashouri,O. T. Inan. Improving the accuracy of proximal timing detection from ballistocardiogram signals using a high bandwidth force plate. Biomedical and Health Informatics (BHI), 2016. Int. Conf. IEEE. 2016: 533-6.

[11] C. Woody. Characterization of an Adaptive Filter for the Analysis of Variable Latency Neuroelectric Signals. Med. Biol. Eng. 1967; 5(6) 539-54.

Address for correspondence:

Ramon Casanella

EETAC-UPC

c/Esteve Terradas 7

08860 Castelldefels (Spain)

ramon.casanella@upc.edu 